\title{
Olfactory Dysfunction in COVID-19 Patients: Findings from a Tertiary Rural Centre
}

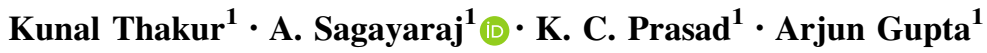

Received: 2 December 2020/Accepted: 4 January 2021/Published online: 18 January 2021

(C) Association of Otolaryngologists of India 2021

\begin{abstract}
Olfactory and/or taste dysfunction are potential neurological manifestations of coronavirus disease -2019 (COVID-19). The aim of the study was to document the prevalence of anosmia in COVID-19 positive patients and analyze the effect of various factors on the occurrence of these chemosensory dysfunction in the local population. Tertiary referral center. Prospective Study. 250 subjects who tested positive for SARS-CoV-2, by real-time polymerase chain reaction (RT-PCR) and admitted in Isolation ward were enrolled for the study. Data was collected from the subjects via oral questionnaire method, based on the AAO-HNS Anosmia Reporting Tool. Data was collected regarding the age, gender, olfactory or gustatory disturbances, history of recent travel or contact with a positive case, smoking, any associated symptoms, any co-morbid conditions and recovery time of sense of olfaction. Out of 250, $179(71.6 \%)$ subjects were diagnosed with Olfactory dysfunction out of which majority were males, 105 $(58.6 \%)$. Most of the patients were above 40 years of age ( $n=184,73.6 \%)$. Majority of the individuals (88 patients) had close contact with a positive case in recent past, followed by 67 patients who were health care workers, hence proving that risk of infection increases with exposure. Anosmia was present in $68.5 \%$ of all the 127 non-smokers. $66.4 \%$ had both olfactory as well as gustatory dysfunction whereas 18 patients $(7.2 \%)$ were found to be totally asymptomatic. Mostly patients recovered their sense of smell within 1-2 weeks from the day of onset of anosmia. Presence of olfactory dysfunction of any degree with or
\end{abstract}

A. Sagayaraj

sagayaraj79@gmail.com

1 Department of ENT and HNS, R.L.J.H and RC, Sri Devaraj Urs Medical College, SDUAHER, Kolar, India without alteration in taste sensation should raise a suspicion of COVID-19 infection, especially when other classical signs are not present. In such conditions, swabs should be sent for confirmation by RT-PCR testing and till results are awaited, the individual should be shifted to quarantine facilities or advised strict self-isolation.

Keywords COVID-19 - SARS-CoV-2 .

Olfactory dysfunction - Gustatory dysfunction .

Chemosensory $\cdot$ Anosmia $\cdot$ Dysgeusia

\section{Introduction}

During the initial phase of COVID-19 disease, the presenting complaints of the patients were mostly fatigue, fever, dry or productive cough, shortness of breath, chest compression, myalgia, diarrhea, vomiting, anorexia, headache, sore throat, dizziness, palpitations, and chest pain.

Over a past few weeks, a significant number of patients are presenting to the Out-patient departments all over the world with complains of loss of smell and/or taste and no other symptoms indicative of severe acute respiratory syndrome-coronavirus 2 (SARS-CoV-2) infection [1].

This has led to an increase in diagnosis of Olfactory dysfunction ranging from anosmia to hyposmia [mostly with some degree of gustatory dysfunction (ageusia or hypogeusia or dysgeusia)], which has coincided with the current COVID-19 pandemic. The Center for Disease Control (CDC), USA now recognizes these as a major sign of coronavirus disease-2019 (COVID-19), the disease caused by this virus [2].

The reports of prevalence of COVID-19-related smell dysfunction and the estimates of recovery rate of sense of olfaction, are highly variable [3]. 
Also, there has been an increase in the scientific studies from many centers across the world about the noticeable rise in the number of cases presenting with loss of smell alone [4].

\section{Materials and Methods}

A prospective study was carried out involving 250 COVID19 positive patients, admitted in the COVID Isolation Ward of our hospital during the September-October months this year. The study was started following approval from the institutional ethical committee, and obtaining informed consent from the subjects.

The data that were assessed included patient age, gender, onset of anosmia, other symptoms, comorbidities, smoking status, any gustatory complaints and the recovery time of olfaction.

All the data were obtained by oral questionnaire method, based on the COVID-19 Anosmia Reporting Tool, developed by the AAO-HNS (American Academy of Otolaryngology-Head and Neck Surgery) [5, 6].

The following inclusion criteria were considered: Adults (> 20 years old); Laboratory-confirmed COVID-19 infection (Reverse Transcription Polymerase Chain Reaction, RT-PCR); and Patients clinically able to fulfill the questionnaire. The exclusion criteria considered were: patients with olfactory or gustatory dysfunctions before the epidemic; patients without a laboratory-confirmed COVID-19 infection diagnosis and patients from the intensive-care unit.

\section{Statistical Methods}

Statistical Package for the Social Sciences for Windows (SPSS version 22,0; IBM Corp, Armonk, NY, USA) was used to perform the statistical analyses. The potential associations between epidemiological, clinical and olfactory and gustatory outcomes were assessed through crosstab generation between two variables (binary or categorical variables) and Chi-square test. A level of $p \leq 0.05$ was used to determine statistical significance.

\section{Analysis and Results}

Following the inclusion and exclusion criteria, 250 patients were enrolled for the study conducted at R.L Jalappa Hospital and Research Center, Kolar. The data obtained from all the subjects are presented in Table 1. Of all the patients enrolled in the study, there were 144 males $(57.6 \%)$ and 106 females $(42.4 \%)$ and majority of them (87 out of 250) were in the age group of 41-50 years.
It was found that 44 patients had no associated risk factors, whereas the rest 206 were associated with one of the three risk factors, considered in the questionnaire. Most people gave history of close contact $(35.2 \%), 26.8 \%$ were health care workers $(26.8 \%)$ while the rest $20.4 \%$ gave history of recent travel to an endemic place. Asthenia was the most common symptom $(52.8 \%)$ which the patients presented with, Headache $(49.6 \%)$ was the second most common, followed by cough which was seen in $43.2 \%$. Rhinorrhea was the least reported symptom $(6 \%)$ in the study subjects.

As can be seen from Fig. 1, Among the 179 patients who developed olfactory dysfunction, $49.6 \%$ of them already had anosmia prior to diagnosis of COVID-19, whereas the rest $22 \%$ developed it after becoming positive for corona virus infection.

Anosmia was present in $68.5 \%$ of all the 127 nonsmokers, while only 4 patients who were heavy smokers (> 30 cigarettes/day) were found to have anosmia. $66.4 \%$ had both olfactory as well as gustatory dysfunction whereas 18 patients $(7.2 \%)$ were found to be totally asymptomatic (Fig. 2).

Mostly patients recovered their sense of smell within 1-2 weeks from the day of onset of anosmia (Fig. 3).

\section{Discussion}

COVID-19 is a form of upper respiratory tract infection, the clinical course of which is still less known and hence is a subject of many webinars and ongoing research projects.

The pathophysiology of the disease and the clinical presentation have a wide variation, so there is an urgent need especially in the developing countries for identification of the most initial and/or alarming symptom, to aid in restricting transmission.

In the early phases of the outbreak, fever, dyspnea, cough, and travel to the high-risk areas (areas declared endemic to the disease) were used as the main screening parameters. However, through the course of the outbreak, various symptoms, like headache, sore throat, nasal congestion, rhinorrhea, fatigue, tonsil swelling, and conjunctivitis, began to be reported from various parts of the world [7].

In our center, Asthenia (52.8\%) was the most presenting symptom, followed by headache $(49.6 \%)$ and cough $(43.2 \%)$. Also, there were 65 patients $(26 \%)$ who had no other associated symptoms (Table 1, Fig. 4).

As the pandemic progressed, chemosensory involvement, in the form of smell and taste dysfunctions, also began to be reported. Many authors reported an increase in the complaints of loss of smell in otherwise asymptomatic patients of anosmia in COVID-19 subjects. After initial 
Table 1 An overview of the distribution of participants according to clinical profile study $(\mathrm{n}=250)$

\begin{tabular}{|c|c|c|}
\hline & Frequency & Percent \\
\hline \multicolumn{3}{|l|}{ Age group in years } \\
\hline $21-30$ & 10 & 4.0 \\
\hline $31-40$ & 56 & 22.4 \\
\hline $41-50$ & 87 & 34.8 \\
\hline $51-60$ & 48 & 19.2 \\
\hline $61-70$ & 34 & 13.6 \\
\hline $71-80$ & 15 & 6.0 \\
\hline \multicolumn{3}{|l|}{ Gender } \\
\hline Male & 144 & 57.6 \\
\hline Female & 106 & 42.4 \\
\hline \multicolumn{3}{|l|}{ Risk factors } \\
\hline None & 44 & 17.6 \\
\hline Health care worker & 67 & 26.8 \\
\hline Close contact with positive case & 88 & 35.2 \\
\hline Travel history & 51 & 20.4 \\
\hline \multicolumn{3}{|l|}{ Onset of anosmia } \\
\hline Before ENT symptoms & 44 & 17.6 \\
\hline After ENT symptoms & 77 & 30.8 \\
\hline At the same time & 58 & 23.2 \\
\hline No symptoms & 65 & 26.0 \\
\hline Cough & 108 & 43.2 \\
\hline Fever & 73 & 29.2 \\
\hline Sore throat & 45 & 18.0 \\
\hline Shortness of breath & 25 & 10.0 \\
\hline Headache & 124 & 49.6 \\
\hline Nasal congestion & 58 & 23.2 \\
\hline Rhinorrhoea & 15 & 6.0 \\
\hline Asthenia & 133 & 52.8 \\
\hline Myalgia & 98 & 39.6 \\
\hline Nausea/vomiting & 23 & 7.6 \\
\hline Diarrhoea & 30 & 10.4 \\
\hline \multicolumn{3}{|l|}{ Addiction } \\
\hline Non-smoker & 127 & $50.8 \%$ \\
\hline Mild smoker & 74 & $29.6 \%$ \\
\hline Moderate smoker & 42 & $16.8 \%$ \\
\hline Heavy smoker & 7 & $2.8 \%$ \\
\hline \multicolumn{3}{|l|}{ Co-morbidities } \\
\hline None & 65 & 26.0 \\
\hline Type 2 diabetes & 15 & 6.0 \\
\hline HTN & 39 & 15.6 \\
\hline Chronic rhinosinusitis & 26 & 10.4 \\
\hline Allergic rhinitis & 47 & 18.8 \\
\hline Asthma & 23 & 9.2 \\
\hline Respiratory complaints & 17 & 6.8 \\
\hline Hepatic dysfunctions & 5 & 2.0 \\
\hline Renal dysfunction & 6 & 2.4 \\
\hline CVS dysfunction & 3 & 1.2 \\
\hline Others & 4 & 1.6 \\
\hline
\end{tabular}

Table 1 continued

\begin{tabular}{lcc}
\hline & Frequency & Percent \\
\hline Gustatory dysfunction & 52 & \\
Not present & 26 & 20.8 \\
Patient not aware & 79 & 10.4 \\
Reduced ability to taste & 93 & 31.6 \\
Distorted taste perception & & 37.2 \\
Recovery time of olfaction & 17 & \\
1-4 days & 87 & 6.8 \\
5-8 days & 103 & 34.8 \\
9-14 days & 43 & 41.2 \\
$>$ 15 days & & 17.2 \\
\hline
\end{tabular}

reports, research studies indicated that anosmia presenting in COVID-19 subjects is more frequent than expected for a routine upper respiratory tract infection [8-10].

In the ongoing pandemic, several studies have reported the presence of smell and taste alterations to be a frequent clinical feature of the coronavirus disease 19 (COVID-19), with a frequency ranging from 19.4 to $88 \%$ of patients [11-13].

Of all the confirmed covid-19 patients admitted in the isolation ward of our hospital, 179 patients (71.6\%) developed olfactory dysfunction whereas 71 patients (28.4\%) did not have any. Among those who developed olfactory dysfunction, $49.6 \%$ of them already developed anosmia prior to diagnosis of COVID-19, whereas the rest $22 \%$ developed it after becoming positive for corona virus infection (Table 2, Fig. 1).

Majority of the patients were above the age of 40 years. $72.9 \%$ of male patients and $69.8 \%$ of female patients had olfactory dysfunction, however this association between Olfactory Dysfunction and gender was not statistically significant (Fig. 2). Around $24 \%$ patients developed anosmia before any other symptoms, $43.01 \%$ after other symptoms while the rest $32.4 \%$ informed that they became aware of loss of smell only when they developed other symptoms (Table 3, Fig. 1). However, no significant correlation was detected between the presence of Olfactory dysfunction and the onset of the same.

Also, the analysis of Olfactory dysfunction with comorbidities and with smoking status was found to be statistically not significant (Table 4).

Recent COVID-19 case series reports are showing a high rate of recovery of olfactory function within 1-2 weeks after the onset of the dysfunction [14].

In our study except for some patients, almost all recovered from the loss of smell over a period of days to weeks. Majority of the patients recovered their sense of smell within the range of 5-8 days and 9-14 days (from the 
Fig. 1 Pie-chart representation of olfactory dysfunction in patients $(\mathrm{n}=250)$
Olfactory Dysfunction

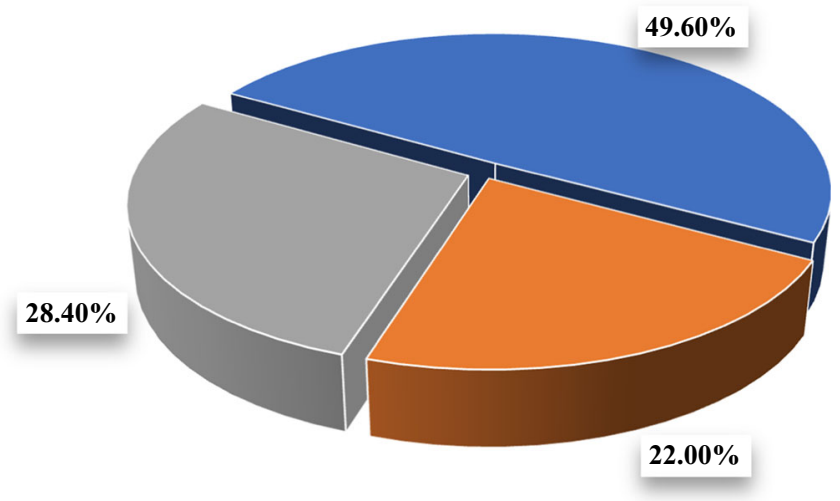

- Developed before diagnosis - Developed after diagnosis " No Olfactory dysfunction

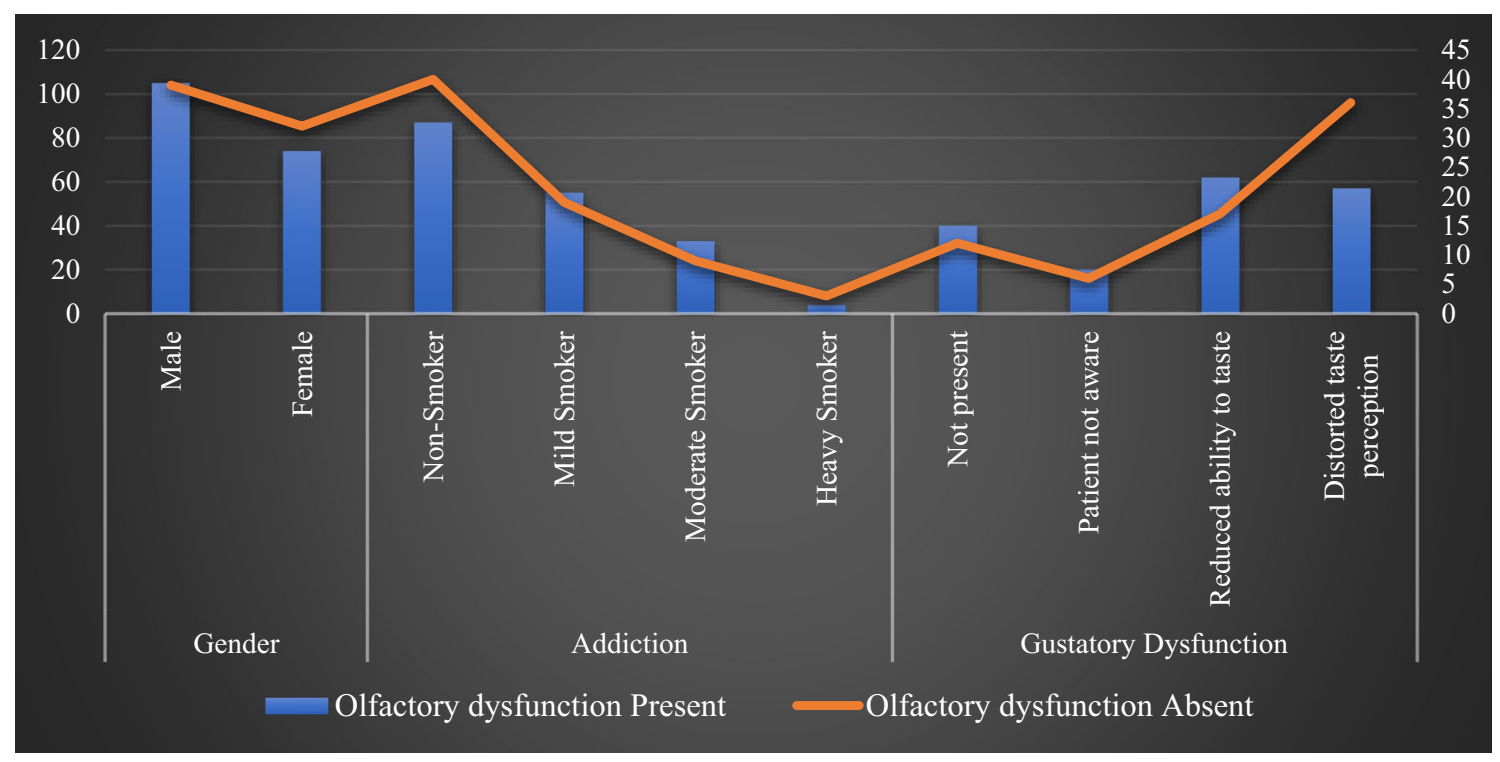

Fig. 2 Graphical representation between various factors and olfactory dysfunction $(n=250)$

day of loss of smell), $36.3 \%$ and $35.7 \%$, respectively. However, in 33 patients the sense of smell did not return to normal during the study time period, so these patients were kept under a separate category (with duration of $>15$ days) (Fig. 3).

The improvement of the chemoceptive function over time, clearly suggests a competitive action of the virus on the receptors of the olfactory and gustatory cells or local inflammatory phenomena, rather than permanent cell damage [15].

There can be a positive co-relation between the viral load and the occurrence or severity of these chemo-sensitive disorders. However, further large-scale studies are needed to investigate about the exact pathogenesis of SARS-CoV-2 in causing these dysfunctions.
Till date many studies have been carried to understand the mechanisms that underlie the loss of olfaction or gustation. Some authors mention that the viral invasion of a variety of olfactory epithelial cell types, which have high expression of ACE2 (Angiotensin-converting-Enzyme type -2 , the main host cell receptor of SARS-CoV-2) and TMPRSS2 (a cell surface protease involved in SARSCoV2 cell entry) can lead to anosmia. It has also been shown that, the target of the virus may not be the neurons but other non-neuronal cells that express ACE2 receptors such as the olfactory epithelium sustentacular cells, microvillar cells, Bowman's gland cells, horizontal basal cells, and olfactory bulb pericytes. Brann et al. speculated that the loss of smell reported by COVID-19 patients is due to the infection of the supporting cells and vascular pericytes of the olfactory epithelium and bulb, which consequently alters the 


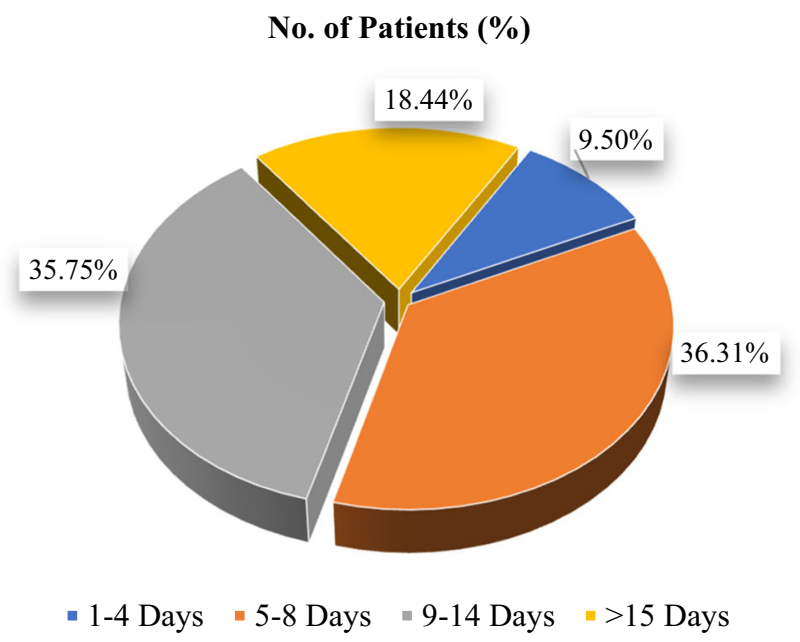

Fig. 3 Representation of recovery time of sense of smell, $n=179$

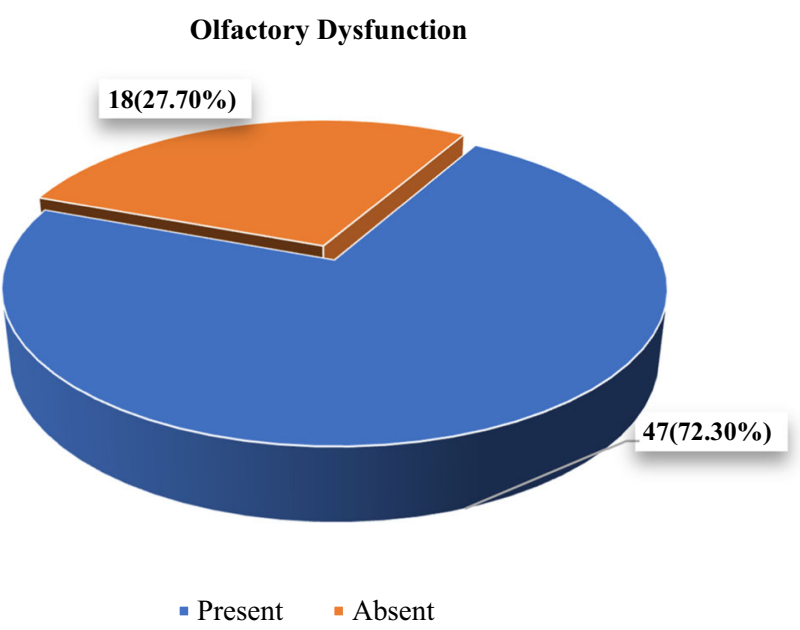

Fig. 4 Representation of patients without any symptom with/without olfactory dysfunction $(n=65)$

function of the olfactory neurons. The further involvement of stem cells (which express lower levels of ACE2 receptors) could be the basis of the long-lasting olfactory dysfunctions [16].

Also, some of the olfactory dysfunction can be conductive-secondary to nasal congestion, swelling, or
Table 3 Description of onset of anosmia in patients

\begin{tabular}{ll}
\hline Onset of anosmia & Olfactory dysfunction present \\
\hline Before other symptoms & $44(24.58 \%)$ \\
After other symptoms & $77(43.01 \%)$ \\
At the same time & $58(32.4 \%)$ \\
Total & 179
\end{tabular}

inflammation preventing olfactory molecules from reaching the olfactory cleft-rather than sensorineural. This may be particularly true in patients who show improvement of their olfactory dysfunction on follow-up, although further research is needed. ACE2 is also highly expressed on the oral mucosa and tongue, representing a potential mechanism for gustatory dysfunction [17].

In our analysis of 179 patients with olfactory dysfunction, $22.3 \%$ had no gustatory dysfunction, $11 \%$ could not notice any difference in taste perception, $34.6 \%$ informed that they had reduced ability to taste whereas the remaining $31.8 \%$ told that they perceived a different taste (Fig. 2), and this association was found to be statistically significant $(p<0.05)$.

We found that around $72.3 \%$ of our patients had anosmia as the only presenting complain, while there were 18 patients $(27.70 \%)$ who had no symptoms, i.e. they did not have anosmia or any other associated symptom. These patients were Asymptomatic throughout, and comprised the $7.2 \%$ of all COVID-19 positive patients enrolled for the study (Table 5, Fig. 4).

So, the need to enquire about "new or sudden loss of smell and/or taste" becomes more evident as, it can be the first and the only symptom in many patients. Also, early identification of individuals who have olfactory dysfunctions with no other symptoms, will help to make the quarantine/isolation measures more effective [18], especially at this stage when there is no specific treatment or vaccine available.

Table 2 Analysis of patients with olfactory dysfunction

\begin{tabular}{lcccc}
\hline Olfactory dysfunction & Frequency & Percent $(\%)$ & Valid percent $(\%)$ & Cumulative percent $(\%)$ \\
\hline Developed before diagnosis & 124 & 49.6 & 49.6 & 49.6 \\
Developed after diagnosis & 55 & 22.0 & 22.0 & 71.6 \\
No olfactory dysfunction & 71 & 28.4 & 28.4 & 100.00 \\
Total & 250 & 100.00 & 100.00 & \\
\hline
\end{tabular}


Table 4 Association between various factors and olfactory dysfunction

\begin{tabular}{lll}
\hline & Olfactory dysfunction & \\
\cline { 2 - 3 } & Present & Absent \\
\hline Gender & & \\
Male & $105(72.9 \%)$ & $39(27.1 \%)$ \\
Female & $74(69.8 \%)$ & $32(30.2 \%)$ \\
Addiction & & \\
Non-smoker & $87(68.5 \%)$ & $40(31.4 \%)$ \\
Mild smoker & $55(74.3 \%)$ & $19(25.6 \%)$ \\
Moderate smoker & $33(78.5 \%)$ & $9(21.4 \%)$ \\
Heavy smoker & $4(57.1 \%)$ & $3(42.8 \%)$ \\
Gustatory dysfunction & & $12(23.1 \%)$ \\
Not present & $40(76.9 \%)$ & $6(23.1 \%)$ \\
Patient not aware & $20(76.9 \%)$ & $17(21.5 \%)$ \\
Reduced ability to taste & $62(78.5 \%)$ & $36(38.7 \%)$ \\
Distorted taste perception & $57(61.3 \%)$ & 0.459 \\
\hline
\end{tabular}

Table 5 Representation of patients without any symptom with/ without olfactory dysfunction

\begin{tabular}{llll}
\hline \multirow{2}{*}{$\begin{array}{l}\text { Total patients without any } \\
\text { symptoms }\end{array}$} & \multicolumn{2}{c}{ Olfactory dysfunction } & $p$ value \\
\cline { 2 - 3 } & Present & Absent & \\
\hline 65 & 47 & 18 & 0.883 \\
& $(72.3 \%)$ & $(27.7 \%)$ & \\
\hline
\end{tabular}

\section{Conclusion}

To break the chain of transmission and flatten the pandemic curve, early identification and isolation of the infected individuals are the two most important measures being undertaken across the world now. With increase in reports of Olfactory and/or gustatory dysfunction, these can be used as important screening criteria for identifying otherwise asymptomatic or atypically symptomatic patients missed by other screening measures.

\section{Recommendation}

Individuals with new onset loss of smell can be considered positive for COVID-19 infection until proven otherwise by a RT-PCR test. Also, the questions related to olfactory and gustatory changes should be incorporated in routine screening measures at all the centers to help identify infected patients prior to further disease transmission.

\section{References}

1. Moein ST, Hashemian SMR, Tabarsi P, Doty RL (2020) Prevalence and reversibility of smell dysfunction measured psychophysically in a cohort of COVID-19 patients. Int Forum Allergy Rhinol 10:1127-1135

2. Al-Ani RM, Acharya D (2020) Prevalence of anosmia and ageusia in patients with COVID-19 at a Primary Health Center, Doha, Qatar. Indian J Otolaryngol Head Neck Surg 19:1-7

3. Sayin I, Yaşar KK, Yazici ZM (2020) Taste and smell impairment in COVID-19: an AAO-HNS anosmia reporting tool-based comparative study. Otolaryngol Head Neck Surg 163:473-479

4. von Bartheld CS, Hagen MM, Butowt R (2020) Prevalence of chemosensory dysfunction in COVID-19 patients: a systematic review and meta-analysis reveals significant ethnic differences. ACS Chem Neurosci 11(19):2944-2961

5. Lao WP, Imam SA, Nguyen SA (2020) Anosmia, hyposmia, and dysgeusia as indicators for positive SARS-CoV-2 infection. World J Otorhinolaryngol Head Neck Surg 6:22-25

6. Kaye R, Chang CWD, Kazahaya K, Brereton J, Denneny JC (2020) COVID-19 anosmia reporting tool: initial findings. Otolaryngol Head Neck Surg 163:132-134

7. Qiu C, Cui C, Hautefort C, Haehner A, Zhao J, Yao Q, Zeng H, Nisenbaum EJ, Liu L, Zhao Y, Zhang D (2020) Olfactory and gustatory dysfunction as an early identifier of COVID-19 in adults and children: an international multicenter study. medRxiv

8. Hopkins C, Surda P, Kumar N (2020) Presentation of new onset anosmia during the COVID-19 pandemic. Rhinology 58(3):295-298

9. Lechner M, Chandrasekharan D, Jumani K, Liu J, Gane S, Lund VJ, Philpott C, Jayaraj S (2020) Anosmia as a presenting symptom of SARS-CoV-2 infection in healthcare workers - a systematic review of the literature, case series, and recommendations for clinical assessment and management. Rhinology 58(4):394-399

10. Lee Y, Min P, Lee S, Kim SW (2020) Prevalence and duration of acute loss of smell or taste in COVID-19 patients. J Korean Med Sci 35(18):e174 
11. Vaira LA, Salzano G, Deiana G, Salzano FA, De Riu G (2020) In response to: in reference to anosmia and ageusia common findings in COVID-19 patients. Laryngoscope 130(9):E506

12. Lechien JR, Chiesa-Estomba CM, De Siati DR et al (2020) Olfactory and gustatory dysfunctions as a clinical presentation of mild-to-moderate forms of the coronavirus disease (COVID-19): a multicenter European study. Eur Arch Otorhinolaryngol 277(8):2251-2261

13. Yan CH, Faraji F, Prajapati DP, Boone CE, DeConde AS (2020) Association of chemosensory dysfunction and COVID-19 in patients presenting with influenza-like symptoms. Int Forum Allergy Rhinol 10:806-813

14. Vaira LA, Deiana G, Fois AG, Pirina P, Madeddu G, De Vito A, Babudieri S, Petrocelli M, Serra A, Bussu F, Ligas E (2020) Objective evaluation of anosmia and ageusia in COVID-19 patients: Single-center experience on 72 cases. Head Neck 42(6): 1252-1258

15. Liu Y, Yang Y, Zhang C, Huang F, Wang F, Yuan J, Wang Z, Li J, Li J, Feng C, Zhang Z (2020) Clinical and biochemical indexes from 2019-nCoV infected patients linked to viral loads and lung injury. Sci China Life Sci 63(3):364-374

16. Brann DH, Tsukahara T, Weinreb C, Logan DW, Datta SR. Nonneural expression of SARS-CoV-2 entry genes in the olfactory system suggests mechanisms underlying COVID-19-associated anosmia. BioRxiv

17. Giacomelli A, Pezzati L, Conti F, Bernacchia D, Siano M, Oreni L, Rusconi S, Gervasoni C, Ridolfo AL, Rizzardini G, Antinori S (2020) Self-reported olfactory and taste disorders in patients with severe acute respiratory coronavirus 2 infection: a cross-sectional study. Clin Infect Dis

18. Beltrán-Corbellini Á et al (2020) Acute-onset smell and taste disorders in the context of COVID-19: a pilot multicentre polymerase chain reaction based case-control study. Eur J Neurol 27(9):1738-1741

Publisher's Note Springer Nature remains neutral with regard to jurisdictional claims in published maps and institutional affiliations. 